\title{
GMR
}

\section{Clinical features of Mexican patients with Mucopolysaccharidosis type I}

\author{
A. Alonzo-Rojo ${ }^{1,4}$, J.E. García-Ortiz ${ }^{1}$, M. Ortiz-Aranda ${ }^{3}$, \\ M.P. Gallegos-Arreola ${ }^{2}$ and L.E. Figuera-Villanueva ${ }^{1,4}$ \\ ${ }^{1}$ Genetics Department, Western Biomedical Research Center, \\ Instituto Mexicano del Seguro Social, Guadalajara, Jalisco, México \\ ${ }^{2}$ Molecular Medicine Department, Western Biomedical Research Center, \\ Instituto Mexicano del Seguro Social, Guadalajara, Jalisco, México \\ ${ }^{3}$ Unidad Médica de Alta Especialidad Hospital de Pediatría, \\ Instituto Mexicano del Seguro Social, Guadalajara, Jalisco, México \\ ${ }^{4}$ Centro Universitario de Ciencias de la Salud, Universidad de Guadalajara, \\ Guadalajara, Jalisco, México
}

Corresponding author: L.E. Figuera-Villanueva

E-mail: luisfiguera@yahoo.com

Genet. Mol. Res. 16 (3): gmr16032602

Received May 30, 2016

Accepted August 11, 2017

Published September 21, 2017

DOI http://dx.doi.org/10.4238/gmr16032602

Copyright $(2017$ The Authors. This is an open-access article distributed under the terms of the Creative Commons Attribution ShareAlike (CC BY-SA) 4.0 License.

\begin{abstract}
Mucopolysaccharidosis type I (MPS-I) is an autosomal recessive lysosomal storage disorder caused by a deficiency or absence of $\alpha$-L-iduronidase, which is involved in the catabolism of glycosaminoglycans (GAGs). This deficiency leads to the accumulation of GAGs in several organs. Given the wide spectrum of the disease, MPS-I has historically been classified into 3 clinical subtypes - severe (Hurler syndrome), intermediate (Hurler-Scheie syndrome), and mild (Scheie syndrome) - none of which is determined by residual enzyme activity. Eleven Mexican patients with MPS-I from northwestern México were evaluated. Diagnoses were confirmed through quantification of GAGs in urine and enzyme assay for $\alpha$-L-iduronidase. Regardless of phenotype, all patients had various degrees of infiltrated facies, short
\end{abstract}

Genetics and Molecular Research 16 (3): gmr16032602 
stature, dysostosis multiplex, joint contractures, and corneal opacity typical of the disease. A better understanding of the spectrum of this disease can assist in diagnosis, treatment, and improvement in the quality of life for these patients.

Key words: Mucopolysaccharidosis I; Glycosaminoglycans; $\alpha$-L-Iduronidase; Hurler syndrome; Scheie syndrome; Mexican population

\section{INTRODUCTION}

Mucopolysaccharidosis type I (MPS-I) is an autosomal recessive disease caused by a deficiency of the lysosomal enzyme $\alpha$-L-iduronidase (IDUA; E.C. 3.2.1.76). This deficiency leads to the accumulation of undegraded glycosaminoglycans, mainly dermatan and heparan sulfates, in tissues and their excess excretion in urine (Neufeld and Muenzer, 2001). MPS is a rare, pan-ethnic disease with an incidence of 1/100,000 live births (Meikle et al., 1999). No epidemiological studies of this disease have been carried out in Mexico, and its behavior and predominant mutations in this population remain unknown. MPS-I has been clinically classified into 3 subtypes. Distinguishing one phenotype from another usually requires assessment of 3 aspects: age of onset of symptoms, neurological impairment, and involvement of organs and systems. The severe phenotype is the Hurler syndrome, with early onset and survival of $<10$ years; the Scheie phenotype is the mildest, and it involves mild skeletal deformities, stiff joints, no mental retardation, and normal life expectancy. Hurler-Scheie, an intermediate phenotype, is characterized by moderate mental impairment and life expectancy of $<25$ years. Recently, the term severe MPS-I has been suggested to describe patients with early onset and rapid progression, whereas attenuated MPS-I is the suggested term for the milder phenotype and longer life span during the disease course (Wraith, 1995; Neufeld and Muenzer, 2001). Phenotypic heterogeneity is thought to be caused by various combinations of mutations of the IDUA gene (OMIM 252800), but other possible explanations are nonpathogenic polymorphism, residual enzymatic activity, the presence of alternative substrates, modifying genes, and environmental factors that can contribute to difficulty in understanding genotypephenotype correlation (Scott et al., 1993; Venturi et al., 2002; Pereira et al., 2008).

A typical clinical presentation includes coarse facial features, short stature, hepatosplenomegaly, dysostosis multiplex, corneal clouding, stiff joints, hearing loss, restrictive lung disease, upper airway obstruction, valvular heart disease, communicating hydrocephalus, spinal cord compression, and mental retardation of varying degrees (Wraith, 1995; Nyhan et al., 2005; Vijay and Wraith, 2005). All subtypes of MPS-I have a wide range of enzyme activity levels, from just below the normal lower limit to undetectable. The IDUA gene has been mapped at $4 \mathrm{p} 16.3$. It is approximately $19 \mathrm{~kb}$ and contains 14 exons, and its messenger RNA transcript is $2.3 \mathrm{~kb}$ and encodes a precursor protein of 653 amino acids (Scott et al., 1990). To date, more than 110 mutations (nonsense, missense, splice site, deletions, and insertions) have been identified throughout the gene. Two mutations, W402X and Q70X, are present in more than $60 \%$ of white patients (Bunge et al., 1994; Scott et al., 1990, 1995; Li et al., 2002; Matte et al., 2003; Guven et al., 2008). The available treatment for MPS-I patients is currently hematopoietic stem-cell transplantation before the age of 2 years. Enzyme replacement therapy with recombinant human IDUA reduces and prevents the accumulation of undegraded substrate. It is indicated for the long-term treatment of non-neurological

Genetics and Molecular Research 16 (3): gmr16032602 
manifestations because it ineffectively crosses the blood-brain barrier and has few effects on the central nervous system (Wraith 2005; Wraith et al., 2005; Beck, 2010). The purpose of this study was to describe the epidemiological aspects and most common clinical manifestations of a group of Mexican patients with MPS-I.

\section{MATERIAL AND METHODS}

Our sample included 11 patients with MPS-I (median age 8.6 years; 3 males and 8 females). Six had the severe phenotype (Hurler syndrome) and 5 had the attenuated phenotype (4 Hurler-Scheie and 1 Scheie). Clinical classifications were based on the age of onset, neurological damage, and involvement of organs and systems. The patients were studied over 2 years (February 2008 to June 2010). MPS-I was suspected based on clinical and radiological features and confirmed with excessive urinary excretion of glycosaminoglycan (GallegosArreola et al., 2000) and enzyme assay deficiency for IDUA using the dried blood spot test. A medical history, complete physical examination, laboratory testing, and X-ray studies were obtained for all patients, and each was assessed using the clinical criteria of the latest Mexican MPS consensus (Grupo de Consenso de la Mucopolisacaridosis Tipo I, 2008).

\section{RESULTS}

The average age of symptom onset was 12 months (range, 2-36 months); for patients with the severe phenotype, the average was 7 months (range, 2-12 months), and for those with the attenuated phenotype, the average was 1.6 years (range, 3-36 months). The earliest diagnosis was made at 4 months in a patient with the severe phenotype and a history of an affected sibling. Diagnosis within the 1st year of life occurred in $27 \%(3 / 11)$; for patients with the severe phenotype, the average age of diagnosis was 2.1 years (range, 4-58 months), whereas that for patients with the attenuated phenotype (5/11) was 7.6 years (range, 12-192 months). The demographic and clinical features of the patients are shown in Tables 1 and 2.

\section{Table 1. Characteristics of the patients with MPS-I.}

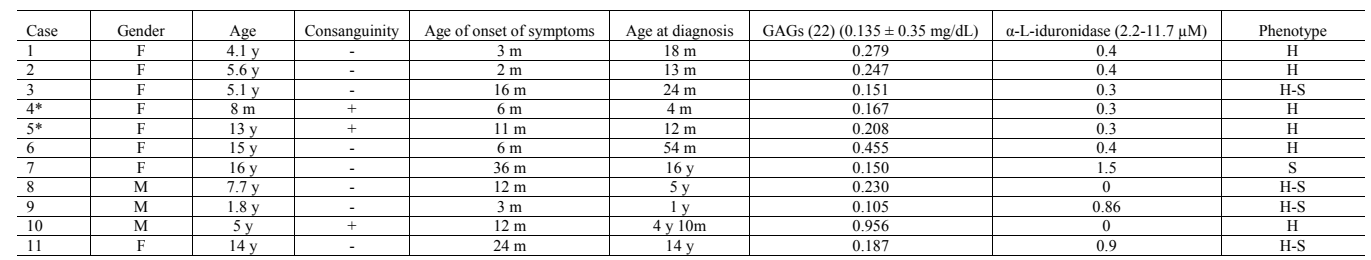

(*) sisters, +: present, m: months, y: years, GAGs: glycosaminoglycans, H: Hurler, H-S Hurler-Scheie, S: Scheie.

\section{Clinical presentation}

Facial dimorphism was a constant feature in patients with the severe MPS-I phenotype: infiltrated coarse features were present in $100 \%$ of patients with various degrees of severity. Short stature was present in $91 \%$, and $27 \%$ had macrocephaly.

Genetics and Molecular Research 16 (3): gmr16032602 
Table 2. Clinical features of MPS patients.

\begin{tabular}{|c|c|c|c|}
\hline \multirow[t]{2}{*}{ Clinical features } & \multirow{2}{*}{$\begin{array}{c}\text { Severe phenotype } \\
\mathrm{N}=6(\%)\end{array}$} & \multirow{2}{*}{$\begin{array}{c}\text { Attenuated phenotype } \\
\mathrm{N}=5(\%)\end{array}$} & \multirow[t]{2}{*}{ Total $(\mathrm{N}=11)(\%)$} \\
\hline & & & \\
\hline \multicolumn{4}{|l|}{ Physical appearance } \\
\hline Coarse facial features & $6(100)$ & $5(100)$ & $11(100)$ \\
\hline Wide and short thorax & $6(100)$ & $5(100)$ & $11(100)$ \\
\hline Short stature & $6(100)$ & $4(80)$ & $10(91)$ \\
\hline Macrocephaly & $2(33)$ & $1(20)$ & $3(27)$ \\
\hline \multicolumn{4}{|l|}{ Skeletal abnormalities } \\
\hline Dysostosis multiplex & $6(100)$ & $5(100)$ & $11(100)$ \\
\hline Short neck & $6(100)$ & $5(100)$ & $11(100)$ \\
\hline Joint stiffness & $6(100)$ & $5(100)$ & $11(100)$ \\
\hline Claw hand & $6(100)$ & $4(80)$ & $10(91)$ \\
\hline Genu valgum & $6(100)$ & $3(60)$ & $9(82)$ \\
\hline Dorsolumbar kyphosis & $5(83)$ & $1(20)$ & $6(55)$ \\
\hline Femoral dysplasia & $3(50)$ & $3(60)$ & $6(55)$ \\
\hline Scoliosis & $3(50)$ & $1(20)$ & $4(36)$ \\
\hline \multicolumn{4}{|l|}{ Ophthalmologic features } \\
\hline Corneal clouding & $6(100)$ & $5(100)$ & $11(100)$ \\
\hline Visual disability & $3(50)$ & $1(20)$ & $4(36)$ \\
\hline Glaucoma & $3(27)$ & 0 & $3(27)$ \\
\hline Megalocornea & $1(17)$ & 0 & $1(9)$ \\
\hline \multicolumn{4}{|l|}{ Respiratory and otolaryngologic feacture } \\
\hline Snoring & $6(100)$ & $4(100)$ & $10(91)$ \\
\hline Chronic rhinitis and/or sinusitis & $5(83)$ & $3(60)$ & $8(73)$ \\
\hline Adenotonsillar hypertrophy & $5(83)$ & $2(40)$ & $7(64)$ \\
\hline Sleep apnea & $5(83)$ & $2(40)$ & $7(64)$ \\
\hline Chronic otitis media & $4(67)$ & $2(40)$ & $6(55)$ \\
\hline Sensorial, conductive or mixed hearing loss & $3(50)$ & $3(60)$ & $6(55)$ \\
\hline Wheeze & $4(67)$ & $1(20)$ & $5(45)$ \\
\hline Sinobronquial syndrome & $1(17)$ & 0 & $1(9)$ \\
\hline Bronchial hyperreactivity & $1(17)$ & 0 & $1(9)$ \\
\hline \multicolumn{4}{|l|}{ Gastrointestinal features and abdominal wall } \\
\hline Hepatomegaly & $6(100)$ & $3(60)$ & $9(82)$ \\
\hline Splenomegaly & $5(83)$ & $1(20)$ & $6(55)$ \\
\hline Umbilical and inguinal hernia & $5(83)$ & $3(60)$ & $8(73)$ \\
\hline Chronic diarrhea & $2(33)$ & $2(40)$ & $4(36)$ \\
\hline Recurrent hernia & $4(67)$ & 0 & $4(36)$ \\
\hline Diastasis recti & $2(33)$ & 0 & $2(18)$ \\
\hline \multicolumn{4}{|l|}{ Cardiac features } \\
\hline Healthy heart & $4(67)$ & $4(80)$ & $8(73)$ \\
\hline Valvular disease & $2(33)$ & $1(20)$ & $3(27)$ \\
\hline \multicolumn{4}{|l|}{ Neurological features } \\
\hline Mental retardation & $6(100)$ & $1(20)$ & $7(64)$ \\
\hline Hydrocephaly & $4(67)$ & 0 & $4(36)$ \\
\hline Attention deficit disorder & $2(33)$ & 0 & $2(18)$ \\
\hline Progressive compression of the spinal cord & $1(17)$ & 0 & $1(9)$ \\
\hline Increased intracranial pression & $1(17)$ & 0 & $1(9)$ \\
\hline Epilepsy & $1(17)$ & 0 & $1(9)$ \\
\hline \multicolumn{4}{|l|}{ Dermathologic features } \\
\hline Thick hair & $6(100)$ & $3(60)$ & $9(82)$ \\
\hline Thick skin & $6(100)$ & $2(40)$ & $8(73)$ \\
\hline Hypertrichosis & $5(83)$ & $1(20)$ & $6(55)$ \\
\hline Persistent Mongolian spots & $3(50)$ & $1(20)$ & $4(36)$ \\
\hline
\end{tabular}

\section{Skeletal features}

The musculoskeletal manifestations were the most constant regardless of the phenotype. Dysostosis multiplex, short neck, and joint contractures were present in $100 \%$ of cases. Besides, the characteristic claw hand and genu valgum was observed in $100 \%$ of patients with the severe phenotype. Thoracolumbar kyphosis, recognized as an early feature, was present in $83 \%$ of patients with the severe phenotype. Femoral dysplasia and scoliosis were present in 50 and $60 \%$, respectively, in patients with the severe phenotype, and in 60 and $20 \%$, respectively, of those with the attenuated phenotype.

Genetics and Molecular Research 16 (3): gmr16032602 


\section{Ophthalmic features}

All patients had corneal opacity independent of the phenotype. Thirty-six percent had decreased visual acuity, and 3 cases of glaucoma and 1 case of megalocornea (severe phenotype) were recorded.

\section{Cardiovascular features}

Within the patient group, $73 \%$ were asymptomatic for cardiovascular manifestations. The valvular disease was present in 2 patients with the severe phenotype (33\%) and 1 patient with the attenuated phenotype (20\%).

\section{Respiratory and otolaryngologic features}

Respiratory and otolaryngologic manifestations were more common in patients with the severe phenotype; however, all patients displayed snoring at some time during the disease. Chronic rhinitis or sinusitis, adenotonsillar hypertrophy, and sleep apnea were observed in $83 \%$ of patients with the severe phenotype, chronic rhinitis or sinusitis was present in $60 \%$, and $40 \%$ with the attenuated phenotype had adenotonsillar hypertrophy and sleep apnea. Some degree of hearing loss was present in 55\% (6/11), but none of the subjects was deaf.

\section{Gastrointestinal and abdominal wall features}

Hepatomegaly was present in $82 \%$ and splenomegaly in $55 \%$ of patients. Umbilical and inguinal hernias accompanied both phenotypes $(73 \%)$, whereas chronic diarrhea was observed in $36 \%$.

\section{Neurological features}

All of the patients with the severe phenotype had mental retardation, but only $20 \%$ $(1 / 5)$ of patients with attenuated phenotypes showed mild to moderate mental retardation. The following neurological manifestations were observed only in patients with the severe phenotype: hydrocephalus (67\%), hyperactivity (33\%), and progressive spinal cord compression, intracranial hypertension, and epilepsy (17\%). Although carpal tunnel syndrome has frequently been reported among MPS-I patients, it was absent in our study group.

\section{Dermatological features}

Thick hair and skin were the most common features observed in both phenotypes: $83 \%$ (severe) and $73 \%$ (attenuated). Hypertrichosis was present in $87 \%$ of patients with the severe phenotype and $20 \%$ of those with the attenuated phenotype. The persistence of Mongolian spots was present in $50 \%$ of patients with the severe phenotype and $20 \%$ of patients with the attenuated phenotype.

\section{DISCUSSION}

MPS-I has traditionally been classified into 3 phenotypes: Hurler, Hurler-Scheie,

Genetics and Molecular Research 16 (3): gmr16032602 
and Scheie, but no clinical or biochemical criteria define the conventional boundaries of these classifications. The severe phenotype (Hurler) represents the most serious of all clinical manifestations, including neurologic impairment. Currently, the tendency is to recognize only 2 phenotypes, severe and attenuated, although factors that might predict the clinical course of the disease, including biomarkers, remain elusive. No comprehensive epidemiological studies that establish the incidence of this disease have been conducted in México. The severe phenotype was most frequently observed in this study, as has been reported in other publications (Alif et al., 2000; Cimaz et al., 2006). This predominance can be explained by the apparent clinical features of the severe phenotype; patients with the attenuated phenotype who lack mental retardations are often underdiagnosed. Although MPS-I is an autosomal recessive disease, our study showed that the female gender was predominant in a ratio of 5:1, and in general, the onset of symptoms occurred on average by the age of 1 year. However, the onset of symptoms in the severe phenotype was 8 months, as reported by others (Vijay and Wraith, 2005).

For patients with the attenuated phenotype, the average age of symptom onset was 1.8 years, although the age of onset up to 2.9 years has been reported, with an earlier diagnosis made in $27 \%$ of cases (Vijay and Wraith, 2005). In the same series of patients, Vijay and Wraith (2005) have reported an average age of symptom onset of 7.6 years in the severe phenotype, with an average age of diagnosis of 9.17 years (range 15 months - 40 years). Notably, late diagnosis in that series was related to delays in some patients whose parents did not seek medical attention and ignorance of the disease by the physician. These possibilities are important to take into account because the earlier the diagnosis is made, the sooner the treatment can begin to improve the quality of life.

The clinical manifestations in patients in the present study were similar to those reported in other studies, but the frequencies differed. Physical appearance, a significant aspect of diagnosis, was present in $100 \%$ of patients and included infiltrating facies, short stature, dysostosis multiplex, joint contractures, and corneal opacity. Mental retardation was present in $100 \%$ of patients with the severe phenotype but only $20 \%$ of those with the attenuated phenotype. This feature is among the most important signs to be evaluated. Mental retardation in patients with the attenuated phenotype was mild to moderate. The remaining neurological manifestations were present only in the severe phenotype.

Skeletal abnormalities were among the most constant features, but joint contractures in patients with the attenuated phenotype may be the earliest and most important clinical manifestation (Alif et al., 2000). Corneal opacity, present in 100\% of the patients in this study, may not be a constant feature. Alif et al. (2000) have reported it in $80 \%$ of cases, taking into account that loss of brightness in the eyes may be the first sign of disease. However, other eye abnormalities such as thickening of the sclera and optic nerve sheath, which occur earlier, must be investigated even before additional clinical features appear (Schumacher et al., 2008). Respiratory and otorhinolaryngologic manifestations were more frequent in patients with the severe phenotype because, in addition to airway obstruction, other factors such as macroglossia were involved and craniofacial abnormalities contributed to repetitive pathologies of the upper airway. Visceromegaly was a common clinical sign among MPS-I patients: $82 \%$ had hepatomegaly and $55 \%$ had splenomegaly in addition to umbilical or inguinal hernias, some of which were recurrent in the severe phenotype (67\%). Accumulated substrate on the skin and annexes leads to the skin and hair thickening (100\%) and hypertrichosis (83\%) observed in the severe phenotype. Persistent Mongolian spots, which have frequently been reported in other series, were present in only $36 \%$ of patients in our study.

Genetics and Molecular Research 16 (3): gmr16032602 
Early diagnosis in patients with MPS-I is essential for forecasting quality of life. Therefore, knowing the clinical spectrum of patients with what is referred to as a rare disease is important. Health personnel should be trained for accurate diagnosis. Now that hematopoietic cell transplantation and enzyme replacement therapy have been introduced, the coming years should bring changes in the natural history of the disease. Current therapeutic challenges such as reducing substrate and the use of intrathecal replacement therapy as an effective alternative therapy for central nervous system manifestations are expected to increase the quality of life and life expectancy in these patients. Genetic counseling and identification of carriers are essential in families with a history of the disease.

\section{ACKNOWLEDGMENTS}

We thank Sanofi Genzyme México for support.

\section{REFERENCES}

Alif N, Hess K, Straczek J, Sebbar S, et al. (2000). Mucopolysaccharidosis type I in Morocco: clinical features and genetic profile. Arch. Pediatr. 7: 597-604. https://doi.org/10.1016/S0929-693X(00)80126-8

Beck M (2010). Emerging drugs for lysosomal storage diseases. Expert Opin. Emerg. Drugs 15: 495-507. https://doi.org/ $10.1517 / 14728214.2010 .498580$

Bunge S, Kleijer WJ, Steglich C, Beck M, et al. (1994). Mucopolysaccharidosis type I: identification of 8 novel mutations and determination of the frequency of the two common alpha-L-iduronidase mutations (W402X and Q70X) among European patients. Hum. Mol. Genet. 3: 861-866. https://doi.org/10.1093/hmg/3.6.861

Cimaz R, Vijay S, Haase C, Coppa GV, et al. (2006). Attenuated type I mucopolysaccharidosis in the differential diagnosis of juvenile idiopathic arthritis: a series of 13 patients with Scheie syndrome. Clin. Exp. Rheumatol. 24: 196-202.

Gallegos-Arreola MP, Machorro-Lazo MV, Flores-Martínez SE, Zúñiga-González GM, et al. (2000). Urinary glycosaminoglycan excretion in healthy subjects and in patients with mucopolysaccharidoses. Arch. Med. Res. 31: 505-510. https://doi.org/10.1016/S0188-4409(00)00104-1

Grupo de Consenso de la Mucopolisacaridosis Tipo I (2008). Mucopolisacaridosis I. Gac. Med. Mex. 144: 9-13.

Guven G, Cehreli ZC, Altun C, Sençimen M, et al. (2008). Mucopolysaccharidosis type I (Hurler syndrome): oral and radiographic findings and ultrastructural/chemical features of enamel and dentin. Oral Surg. Oral Med. Oral Pathol. Oral Radiol. Endod. 105: 72-78. https://doi.org/10.1016/j.tripleo.2007.02.015

Li P, Wood T and Thompson JN (2002). Diversity of mutations and distribution of single nucleotide polymorphic alleles in the human alpha-L-iduronidase (IDUA) gene. Genet. Med. 4: 420-426. https://doi.org/10.1097/00125817200211000-00004

Matte U, Yogalingam G, Brooks D, Leistner S, et al. (2003). Identification and characterization of 13 new mutations in mucopolysaccharidosis type I patients. Mol. Genet. Metab. 78: 37-43. https://doi.org/10.1016/S1096-7192(02)00200-7

Meikle PJ, Hopwood JJ, Clague AE and Carey WF (1999). Prevalence of lysosomal storage disorders. JAMA 281: 249254. https://doi.org/10.1001/jama.281.3.249

Neufeld EF and Muenzer J (2001). The Mucopolysaccharidosis. In: Molecular and metabolic bases of inherited disease (Scriver CR, Beaudet AL, Valle D, Sly WS, et al., eds.). McGraw-Hill, New York, 3421-3443.

Nyhan WL, Barshop BA and Ozand PT (2005). Mucopolysaccharidosis. In: Atlas of Metabolic Diseases (Nyhan WL, Barshop BA and Ozand PT, eds.). Oxford University Press, New York, 501-511.

Pereira VG, Martins AM, Micheletti C and D'Almeida V (2008). Mutational and oxidative stress analysis in patients with mucopolysaccharidosis type I undergoing enzyme replacement therapy. Clin. Chim. Acta 387: 75-79. https://doi. org/10.1016/j.cca.2007.09.008

Schumacher RG, Brzezinska R, Schulze-Frenking G and Pitz S (2008). Sonographic ocular findings in patients with mucopolysaccharidoses I, II and VI. Pediatr. Radiol. 38: 543-550. https://doi.org/10.1007/s00247-008-0788-y

Scott HS, Ashton LJ, Eyre HJ, Baker E, et al. (1990). Chromosomal localization of the human alpha-L-iduronidase gene (IDUA) to 4p16.3. Am. J. Hum. Genet. 47: 802-807.

Scott HS, Litjens T, Nelson PV, Thompson PR, et al. (1993). Identification of mutations in the alpha-L-iduronidase gene (IDUA) that cause Hurler and Scheie syndromes. Am. J. Hum. Genet. 53: 973-986.

Genetics and Molecular Research 16 (3): gmr16032602 
Scott HS, Bunge S, Gal A, Clarke LA, et al. (1995). Molecular genetics of mucopolysaccharidosis type I: diagnostic, clinical, and biological implications. Hum. Mutat. 6: 288-302. https://doi.org/10.1002/humu.1380060403

Venturi N, Rovelli A, Parini R, Menni F, et al. (2002). Molecular analysis of 30 mucopolysaccharidosis type I patients: evaluation of the mutational spectrum in Italian population and identification of 13 novel mutations. Hum. Mutat. 20: 231. https://doi.org/10.1002/humu.9051

Vijay S and Wraith JE (2005). Clinical presentation and follow-up of patients with the attenuated phenotype of mucopolysaccharidosis type I. Acta Paediatr. 94: 872-877. https://doi.org/10.1080/08035250510031584

Wraith JE (1995). The mucopolysaccharidoses: a clinical review and guide to management. Arch. Dis. Child. 72: 263-267. https://doi.org/10.1136/adc.72.3.263

Wraith EJ, Hopwood JJ, Fuller M, Meikle PJ, et al. (2005). Laronidase treatment of mucopolysaccharidosis I. BioDrugs 19: 1-7. https://doi.org/10.2165/00063030-200519010-00001

Wraith JE (2005). The first 5 years of clinical experience with laronidase enzyme replacement therapy for mucopolysaccharidosis I. Expert Opin. Pharmacother: 6: 489-506. https://doi.org/10.1517/14656566.6.3.489

Genetics and Molecular Research 16 (3): gmr16032602 